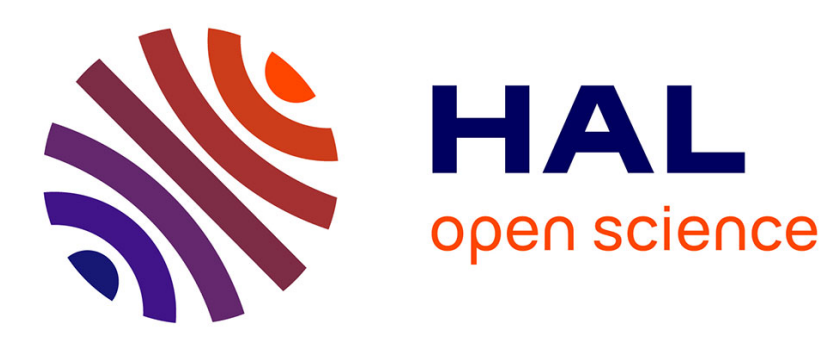

\title{
A numerical assessment of wall shear stress changes after endovascular stenting
}

\author{
Franck Nicoud, Hélène Vernhet, Michel Dauzat
}

\section{To cite this version:}

Franck Nicoud, Hélène Vernhet, Michel Dauzat. A numerical assessment of wall shear stress changes after endovascular stenting. Journal of Biomechanics, 2005, 38 (10), pp.2019-2027. 10.1016/j.jbiomech.2004.09.011 . hal-00908281

\section{HAL Id: hal-00908281 \\ https://hal.science/hal-00908281}

Submitted on 22 Nov 2013

HAL is a multi-disciplinary open access archive for the deposit and dissemination of scientific research documents, whether they are published or not. The documents may come from teaching and research institutions in France or abroad, or from public or private research centers.
L'archive ouverte pluridisciplinaire HAL, est destinée au dépôt et à la diffusion de documents scientifiques de niveau recherche, publiés ou non, émanant des établissements d'enseignement et de recherche français ou étrangers, des laboratoires publics ou privés. 
A Numerical Assessment of Wall Shear Stress Changes

after Endovascular Stenting

\author{
F. Nicoud \\ University Montpellier II - CNRS UMR 5149
}

H. Vernhet \& M. Dauzat

University Montpellier I - Laboratory of Cardiovascular Physiology EA 2992

$\underline{\text { Manuscript number: }}$

BIO/2003/003572 - 2nd revision

Corresponding author:

Prof. F. Nicoud

University of Montpellier II

CC 051

34095 Montpellier Cedex 5

France

tel: $+33(0) 467144846$

fax: $+33(0) 467149316$

E-mail: nicoud@math.univ-montp2.fr

Keywords:

stenting,

restenosis,

wall shear stress,

compliance,

numerical method

Word count:

3300 
Abstract

This theoretical/numerical study aims at assessing the hemodynamic changes induced by endovascular stenting. By using the classical one-dimensional linear pressure waves theory in elastic vessels, we first show that the modulus of the reflection coefficient induced by an endovascular prosthesis is most likely small since proportional to the stent-to-wavelength ratio. As a direct consequence, the wall motion of the elastic (stented) artery can be prescribed a priori and the coupled fluid-structure problem does not have to be solved for assessing the hemodynamic changes due to stenting. Several 2D axisymetric calculations are performed to solve the unsteady incompressible Navier-Stokes equations on moving meshes for different types of (stented) arteries. The numerical results suggest that endovascular stenting increases the systodiastolic variations of the wall shear stress (by $35 \%$ at the middle of the stent, by almost $50 \%$ in the proximal transition region). Additional calculations show that over-dilated stents produce less hemodynamic perturbations. Indeed, the increase of the amplitude of the wall shear stress variations over the cardiac cycle is only $10 \%$ when the stent radius is equal to the radius of the elastic artery at systole (instead of being equal to the mean artery radius). 


\section{Motivation \& Objectives}

Angioplasty with or without endovascular stenting is an established minimally invasive technique that is used as treatment of occlusive disease in medium to large arteries. It has been applied extensively in the coronary, renal, and peripheral vascular systems. The use of intravascular stents tends to lower the complication rate although restenosis rates as high as $15-30 \%$ after 6 months have been observed in human coronary arteries (Rau et al. 1998; Serruys et al. 2002). Coated stents eluting antimitotic agents such as sirolimus and paclitaxel inhibit intimal proliferation. Preliminary reports have showed extremely low rates of restenosis over the short and mid-term (Morice et al. 2002; Grube et al. 2003). However, considering the long-standing changes in wall mechanics induced by stent placement, long term results of coated stents are still required. Besides, a better understanding of the mechanical effects induced by stenting could lead to improved prosthesis with lower failure rates and smaller coating requirements (if any). This study is a contribution to this quest for better stent design.

One possible explanation for the trend to restenosis relies on the hemodynamic modifications induced by the prosthesis. Changes in wall shear stress induce endothelial dysfunction (Caro et al. 1969), ultimately leading to intimal hyperplasia and restenosis. Davies et al. (2001) suggest that the magnitude of the shear stress is of secondary importance to the spatial and temporal fluctuations of this quantity. In vivo testing performed by Rolland et al. (1999); Vernhet et al. (2000, 2001) show that endovascular stenting induces a large modification of arterial compliance and thus may modify the propagation of arterial waves by introducing additional reflections. The first objective of this study is then to assess the amount of pressure wave reflection due to stenting (section 2). Another expected effect of the compliance mismatch induced by stenting is to modify the details of blood motion in the stented area. Specifically, the time-averaged (over the cardiac cycle) wall shear stress might be changed, as well as the level of its systolo-diastolic variations. The second goal of this paper is to clarify the amount of changes in blood motion that can be expected in relation to endovascular stenting (section 3). Previous studies have addressed 
the effects of stenting at a micro-scale level, including the geometrical description of the struts shape and spacing (Berry et al. 2000; Bénard et al. 2003). These studies provide information relevant to the hemodynamic changes immediately after stenting, before the wires have been integrated with the surrounding tissue. For example, the numerical results obtained by Berry et al. (2000) suggest that the flow may reattach downstream of the wires when the strut spacing is greater than about six wire diameters. On the contrary, the present study is dealing with the global effects of the compliance mismatch, neglecting the details of the prosthesis structure. Instead of considering a rigid wall with a micro-scale geometrical description of the struts shape (Berry et al. 2000; Bénard et al. 2003), we model the prosthesis as a uniform elastic tube with its own compliance. The results of this study are more relevant to long-standing stenting, after the wires have been integrated with the surrounding tissue. The reason for considering this point of view is that intimal hyperplasia is certainly a long lasting phenomenon compared to the integration of the stent wires by the vessel: for example, only one week after stenting, Robinson et al. (1988) observed a nearly continuous endothelial and pseudo-endothelial cell layer on the luminal surface of the stented rabbit aorta while intimal hyperplasia is only completed after six 6 to 12 weeks for this model. Therefore, a micro-scale analysis of the blood flow changes including the struts details is only relevant to acute stenting effects. In this paper we consider a complementary point of view in order to provide relevant information about the long-standing hemodynamic effects of stenting.

\section{Pressure waves reflection due to stenting}

\subsection{Basic equations}

The general one-dimensional equations describing the pulsatile blood flow in compliant arteries are well known since the work of Hughes \& Lubliner (1973). They describe the evolution of $u$ and $P$, the averaged (over the cross section of the artery) velocity and relative pressure 
respectively. Following Reuderink et al. (1989), the non-linear terms are often neglected. Furthermore, considering a segment whose cross section area $(A)$ and compliance $\left(A^{\prime}=d A / d P\right)$ do not depend on the space variable $x$ and letting $P=\hat{P} \exp (-j \omega t)$ and $u=\hat{u} \exp (-j \omega t)$, where $j^{2}=-1$ and $\omega$ is the wave pulsation, the following Helmholtz equation can be derived from the classical linear wave equation:

$$
d^{2} \hat{P} / d x^{2}+k^{2} \hat{P}=0
$$

The complex wave number is $k=\sqrt{\omega\left(\rho \omega+j f_{v}\right) A^{\prime} / A}$, where $\rho$ stands for the blood density and $f_{v}$ is related to the viscous drag and can be derived from the Womersley velocity profile (Womersley 1955). The complex wave speed is $c=\omega / k$ and the general solution within a homogeneous segment is :

$$
\hat{P}=P^{+} e^{j k\left(x-x_{0}\right)}+P^{-} e^{-j k\left(x-x_{0}\right)} \quad, \quad \hat{u}=\frac{k}{\rho \omega+j f_{v}}\left(P^{+} e^{j k\left(x-x_{0}\right)}-P^{-} e^{-j k\left(x-x_{0}\right)}\right)
$$

where $x_{0}$ is the axial position of the left boundary of the segment and $P^{+}$and $P^{-}$correspond to the amplitude of the forward and backward pressure waves. Their values are determined in order to satisfy the boundary conditions at $x=x_{0}$ and $x=x_{0}+L$ where $L$ is the length of the segment.

\subsection{Modelling the endovascular stenting}

In the purpose of modelling the wave reflection induced by an endovascular stent placed in an elastic artery, three successive homogeneous segments are considered, each having its own set of constant area and compliance (see figure 1). Each physical quantity in segment number $i(i=1,2,3)$ is denoted with indice $i$. Conservation of the total flow rate and energy at the interfaces $1-2$ and $2-3$ requires, for $j=1,2$ :

$$
A_{j} \hat{u}_{j}\left(x_{0_{j}}+L_{j}\right)=A_{j+1} \hat{u}_{j+1}\left(x_{0_{j+1}}\right) \quad, \quad \hat{P}_{j}\left(x_{0_{j}}+L_{j}\right)=\hat{P}_{j+1}\left(x_{0_{j+1}}\right)
$$

Two boundary conditions at $x=x_{0_{1}}=0$ and $x=x_{0_{3}}+L_{3}$ are needed in order to close the problem. In order to focus on the stent response, non-reflecting boundary conditions are 


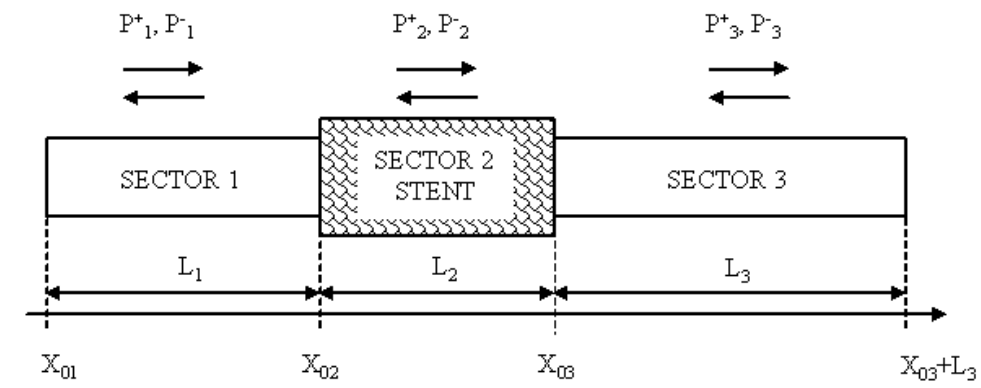

Figure 1. Schematic of the three homogeneous segments used to build a model of stented artery as regards propagation and reflexion.

prescribed at both sides, leading to $P_{1}^{+}=1$ and $P_{3}^{-}=0$. The four remaining wave amplitudes, viz. $P_{2}^{+}, P_{3}^{+}, P_{1}^{-}, P_{2}^{-}$, are determined by solving Eqs. (2.3) for $j=1,2$. The complex coefficient of wave reflection due to the stent is then defined as $R_{\text {stent }}=\exp \left(-2 j k_{1} L_{1}\right) P_{1}^{-} / P_{1}^{+}$. After some algebra one finds out:

$$
R_{\text {stent }}=\frac{A_{2} K_{2}\left(A_{1} K_{1}-A_{3} K_{3}\right) \cos \left(k_{2} L_{2}\right)-j\left(A_{1} K_{1} A_{3} K_{3}-\left(A_{2} K_{2}\right)^{2}\right) \sin \left(k_{2} L_{2}\right)}{A_{2} K_{2}\left(A_{1} K_{1}+A_{3} K_{3}\right) \cos \left(k_{2} L_{2}\right)-j\left(A_{1} K_{1} A_{3} K_{3}+\left(A_{2} K_{2}\right)^{2}\right) \sin \left(k_{2} L_{2}\right)}
$$

where $K_{i}=k_{i} /\left(\rho \omega+j f_{v_{i}}\right)$. This theoretical result will serve as a support of the wall motion used in the numerical study.

\section{Wall shear stress changes due to stenting}

The simple 1D analysis provided in section 2 cannot be used to gain insights about the details of the fluid motion modifications related to artery stenting. Indeed, no reasonable assumption regarding the shape of the velocity profile within the transition area can be formulated a priori and the multi-dimensional Navier-Stokes equations must be solved (assuming Newtonian blood rheology). The solver used in this study is based on the projection method of Chorin (1967) with finite element discretization and Arbitrary Lagrangian Eulerian formulation to handle moving boundaries. This code has been extensively validated by computing classical (Medic \& Mohammadi 1999) as well as blood flow specific test cases (Nicoud 2002). 


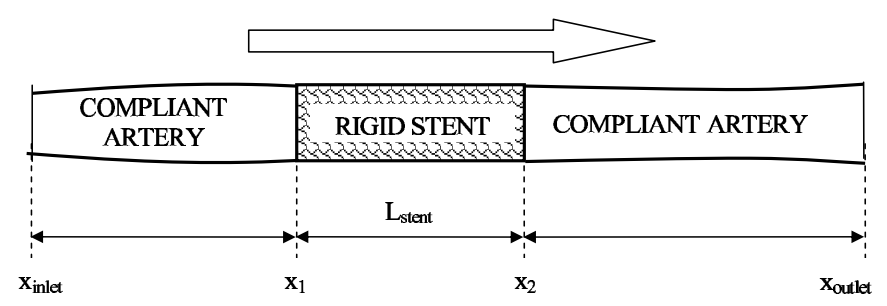

Figure 2. Schematic of the computational domain.

\subsection{Computational domain}

Since our objective is to investigate the global effect of the compliance mismatch induced by stenting, the endovascular prosthesis is modelled as a uniform duct (the details of the struts are not represented) whose wall is not compliant. Such "prosthesis" is inserted within an elastic artery with compliant wall, as depicted in figure 2. The computational domain is sufficiently short to assume constant host artery characteristics and neglect the viscous damping of waves. The flow rate entering the domain is taken as $Q\left(x_{\text {inlet }}, t\right)=Q_{0}+Q_{1} \exp \left(j \omega\left(t-x_{\text {inlet }} / c\right)\right)$, where $\omega$ is the pulsation, $x_{\text {inlet }}$ is the axial position of the inlet section and $Q_{0}$ and $Q_{1}$ stand for the steady and pulsed parts of the flow rate. The mechanical and geometrical data were obtained from animal experimentations performed by Vernhet et al. (2001): the pulsation is $\omega=8 \pi$, the mean artery radius is $R_{0}=1.5 \mathrm{~mm}$, the distensibility coefficient of the nonstented artery is $A^{\prime} / A=20,7 \cdot 10^{-6} \mathrm{~Pa}^{-1}$ and the length of the stent is $L_{\text {stent }}=13 \mathrm{~mm}$. After stenting, acute measurements (Vernhet et al. 2001) show that the compliance at the stent level is approximately three times smaller than in the non-stented vessel. Measurements made three months later (Vernhet et al. 2003) show that long-standing stenting is also responsible for increased compliance values upstream from the stent, resulting in a larger compliance ratio of order 5 to 6 . In any case, the compliance at the stent level is several times smaller than that of the host vessel and has been neglected during this numerical study (rigid prosthesis assumption). Note however that there would be no fundamental issues in accounting for the wall motion at the stent level. 
Actually, the motion of the vessel boundary results from the coupling between the fluid and wall mechanics and the local radius is mostly related to the pressure field. Such a coupling is difficult to handle since the density of blood and tissues are of the same order and because the rheology of the vessels is far from well understood. Under the linear elasticity assumption, Tortoriello \& Pedrizzetti (2004) recently used a perturbative approach in order to replace the coupled fluid-vessel problem by a cascade of two simpler weakly coupled problems. In this approach, the first problem provides the exact solution into a rigid vessel, the second one approximates the blood flow modifications due to the compliant wall. Since the wall shear stress is expected to be very sensitive to the wall motion, we did follow a different approach where the the exact fluid flow equations (and not their truncated linear expansion) are solved for. Since we are mostly interested in the response of the fluid mechanics to wall motion perturbations, the fluid-wall coupling problem can be avoided by prescribing the wall motion a priori. In absence of reflection and in the case of an elastic uniform artery whose mean radius is $R_{0}$, the wall motion induced by a propagative pressure wave of pulsation $\omega$ may be written as:

$$
R(x, t)=R_{0}\left(1+\epsilon e^{j(\omega t-k x)}\right), \quad k=\frac{\omega}{c}
$$

where the wave number $k$ is related to the speed of the (forward) pressure wave $c$. Note that $\epsilon=\max \left(R(x, t)-R_{0}\right) / R_{0}$, the relative amplitude of the radius variations, is a small parameter of the problem: it is zero for a perfectly rigid artery while the animal experiments of Vernhet et al. (2001) suggest $\epsilon \simeq 0.05$. The speed of propagation is fixed by stating that the non-stented artery being uniform along the streamwise direction, the mass flow rate at any section $x=X$ should be the time-lagged version of the mass flow rate at $x=x_{\text {inlet }}$. The conservation of mass applied to the artery sector $x_{\text {inlet }}<x<X$ then implies that (Nicoud 2002):

$$
c=\frac{Q_{1}}{2 A_{0} \epsilon}+O(1), \quad A_{0}=\pi R_{0}^{2}
$$

where $O(1)$ is a term of order unity which can be neglected since $c$ is of order $1 / \epsilon$ according to Eq. (3.2). From the physiological data obtained by Vernhet et al. (2001), the following values 
were used for the flow rate: $Q_{0} \simeq 2413 \mathrm{~mm}^{3} / \mathrm{s}$ and $Q_{1} \simeq 1761 \mathrm{~mm}^{3} / \mathrm{s}$. Eq. (3.2) then leads to $c \simeq 2492 \mathrm{~mm} / \mathrm{s}$. With $\omega=8 \pi$, the corresponding wavelength is $\lambda \simeq 623 \mathrm{~mm}$. In the case where the vessel is stented between $x_{1}$ and $x_{2}$ (see figure 2), the wall displacement is virtually zeroed (fully rigid stent) for $x_{1}<x<x_{2}$ and the following expression is valid for any axial position and time :

$$
R(x, t)=R_{0}\left(1+\epsilon f(x) e^{j(\omega t-k x)}\right)+(1-f(x)) \delta R_{\text {stent }}
$$

where the damping function is $f(x)=\left[1-\tanh \left(x-x_{1}\right)\right] / 2$ for $x<\left(x_{1}+x_{2}\right) / 2$ and $f(x)=$ $\left[1+\tanh \left(x-x_{2}\right)\right] \exp \left(j k\left(x_{2}-x_{1}\right)\right) / 2$ for $x>\left(x_{1}+x_{2}\right) / 2$ and $\delta R_{\text {stent }}=R_{\text {stent }}-R_{0}$ is the amount of overdilation. Note that the term "overdilation" refers here to any case where the stent radius is greater than the mean radius of the vessel before stenting $R_{0}$. This is somewhat different from the clinical practice where the term "overdilation" corresponds to cases where the stent radius (after recoil) is greater than the maximum (systolic) radius of the vessel close to the prosthesis (although the difference between mean and maximum radius can hardly be measured routinely).

\subsection{Numerical results}

Several 2D axisymmetric simulations have been performed based on the computational domain and wall motion described in section 3.1. In all cases, the bulk Reynolds number based on the steady part of the flow rate $Q_{0}$ and the mean radius $R_{0}$ is close to $R_{b}=102$. The Womersley number is $W_{0}=3.36$. The velocity profile is imposed at the inlet section $x=x_{\text {inlet }}$ following the Womersley solution in elastic tubes and a zero constraint condition is used at the outlet section $x=x_{\text {outlet }}$. In order to assess the effect of the inlet/outlet boundary conditions on the results, computational domains with two different lengths have been considered. Two different spatial resolutions were also used in order to assess the spatial discretization errors. The main characteristics of the calculations performed are given in table 1 where $\Delta x$ is the grid spacing in the streamwise direction in the area $x_{1}<x<x_{2}$ and $\Delta r$ refers to the grid spacing in the 
radial direction. Runs $\mathrm{R} 1$ and $\mathrm{R} 2$ correspond to reference calculations without endovascular prosthesis, the artery being fully rigid (no wall displacement) for R1 and elastic for R2. Labels $\mathrm{R} 3(\mathrm{x})$ and R4 correspond to runs with stenting, the overdilation being non zero only for the latter where $\delta R_{\text {stent }}=\epsilon R_{0}$ (the stent radius is equal to the artery radius at systole). Recall that the term "overdilation" refers here to a stent whose radius is greater than $R_{0}$ (see section 3.1). For all the cases considered in table 1, the relative variation of the radius of the stented vessel $\left(\delta R(x, t)-R_{0}\right) / R_{0}$ is less than $5 \%$, which is consistent with the linear elasticity assumption used to derive Eq. (3.1). When present, the stent is between $x_{1}=34 \mathrm{~mm}$ and $x_{2}=47 \mathrm{~mm}$. Runs whose label contains 'a' have been performed with a longer computational domain than others. Labels containing letter 'b' correspond to runs with finer mesh in the streamwise direction. In all cases, four cardiac cycles were first computed in order to reach a periodic state. A fifth cycle was then computed in order to analyze the results and compare the different physical/numerical configurations. Comparisons of the runs R2, R2a and R3, R3a, R3b (not shown) indicate that there is no significant effect of the numerics in the stent region (Nicoud 2002). The differences observed between R1, R2, R3 and R4 are thus relevant to the stenting effects.

Time evolutions of the flow rate at inlet and outlet sections are displayed in figure 3 for the case R2. The constraint that was introduced in section 3.1 in order to set the speed of propagation of the pressure wave is fulfilled satisfactorily. Indeed, the flow rate at $x=x_{\text {outlet }}$ is the signal at $x=x_{\text {inlet }}$ with a time lag close to $\left(x_{\text {outlet }}-x_{\text {inlet }}\right) / c \simeq 80 / 2492 \simeq 0.032 \mathrm{~s}$. In absence of endovascular prosthesis, all the physical quantities are self-similar with the constant speed of propagation $c$ along the computational domain. Due to the wall displacement, Eq. (3.1), the wall shear stress (WSS) is not uniform over the streamwise direction, as depicted in figure 4 at four different instants. Instead it is alternatively increasing and decreasing along the domain depending on the phase considered. In the case where the vessel is not compliant, there should be no time lag between shear stress signals at different locations since the exact Womersley profile is imposed at $x=x_{\text {inlet }}$. Accordingly, the WSS is mostly uniform (not exactly uniform 


\begin{tabular}{|c|c|c|c|c|c|c|c|c|}
\hline Run & Wall motion & $\epsilon$ & $\delta R_{\text {stent }}$ & $x_{\text {inlet }}$ & $x_{\text {outlet }}$ & $\Delta x$ & $\Delta r$ & \# of grid points \\
\hline $\mathrm{R} 1$ & $R(x, t)=R_{0}$ & 0.0 & no stent & 0 & 80 & 0.075 & 0.286 & 3528 \\
\hline $\mathrm{R} 2$ & Eq. (3.1) & 0.05 & no stent & 0 & 80 & 0.075 & 0.286 & 3528 \\
\hline R3 & Eq. (3.3) & 0.05 & 0.0 & 0 & 80 & 0.075 & 0.286 & 3528 \\
\hline $\mathrm{R} 4$ & Eq. (3.3) & 0.05 & 0.075 & 0 & 80 & 0.075 & 0.286 & 3528 \\
\hline $\mathrm{R} 2 \mathrm{a}$ & Eq. (3.1) & 0.05 & no stent & -30 & 110 & 0.075 & 0.286 & 4368 \\
\hline R3a & Eq. (3.3) & 0.05 & 0.0 & -30 & 110 & 0.075 & 0.286 & 4368 \\
\hline $\mathrm{R} 3 \mathrm{~b}$ & Eq. (3.3) & 0.05 & 0.0 & 0 & 80 & 0.050 & 0.286 & 5208 \\
\hline
\end{tabular}

TABLE 1. List of the axisymmetric calculations performed with their main numerical characteristics. Lengths and axial positions are in millimetres.

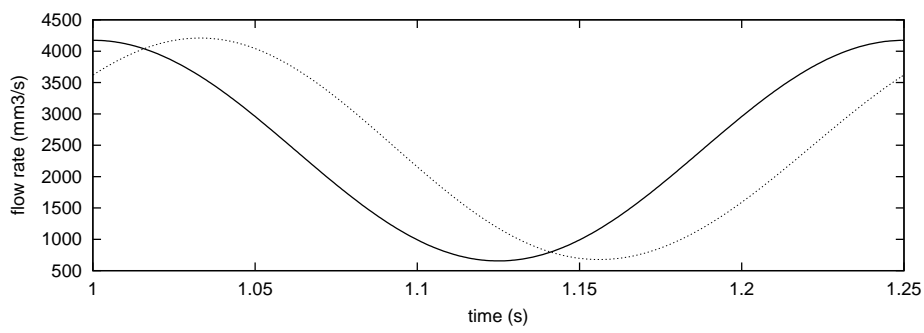

Figure 3 . Time evolutions of the flow rate at sections $-: x=x_{\text {inlet }}$ and $\cdots \cdots \cdots: x=x_{\text {outlet }}$ for the case R2. Note that the origin of time is $1 \mathrm{~s}$ since the fifth cycle is analysed (see text).

because of small side effects due to the inlet/outlet boundary conditions) over the streamwise distance in the case R1 (see figure 4$)$.

The shape of the computational domain for cases R2, R3 and R4 is shown in figure 5 for times $t=n T$ (corresponding to systole at the inlet section) and $t=(n+1 / 2) T$ (diastole). The non-compliant region which mimics the endovascular prosthesis is clearly visible (cases 

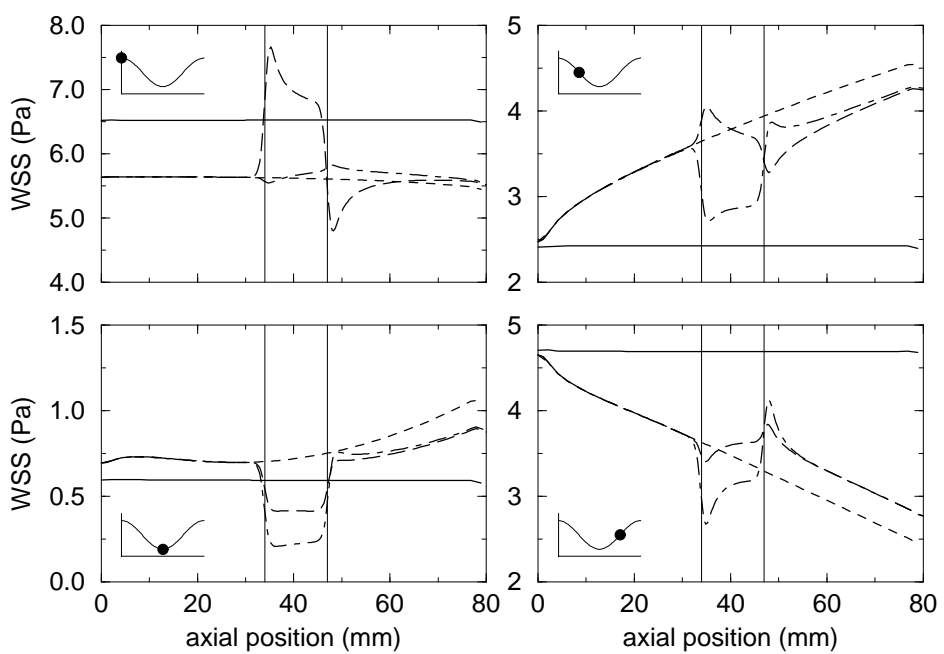

Figure 4. Wall shear stress for runs R1 (solid), R2 (dashed), R3 (long dashed) and R4 (dot-dashed) at times: $t=n T$ (systole - top left), $t=(n+1 / 4) T$ (top right), $t=(n+1 / 2) T$ (diastole - bottom left) and $t=(n+3 / 4) T$ (bottom right), where $T=2 \pi / \omega$ is the period. The two vertical lines denote the stent location.

R3 and R4) in the central region. The isolines of the streamwise velocity show that the flow accelerates when the cross section area decreases. The effect of the wall motion mismatch on the WSS is also shown in figure 4. At systole, this quantity is larger in the medium part of the stented region $(x \simeq 40 \mathrm{~mm})$ than in the non-stented artery when the stent is not overdilated. This is consistent with the fact that the cross section area in R3 and at systole is smaller in the prosthesis zone (see figure 5). On the contrary, when the stent is slightly overdilated, the WSS at systole is not drastically modified by the prosthesis. Indeed, stenting does not induce any geometrical perturbation in this case (see figures 4 and 5, case R4). At diastole, the cross section area at the stent level is larger and the WSS is smaller. Comparing the cases R3 and R4, we note that this effect is amplified by a slight increase of the stent diameter. However, the impact of the stent overdilation on the WSS modification is not as pronounced at diastole than during systole. 

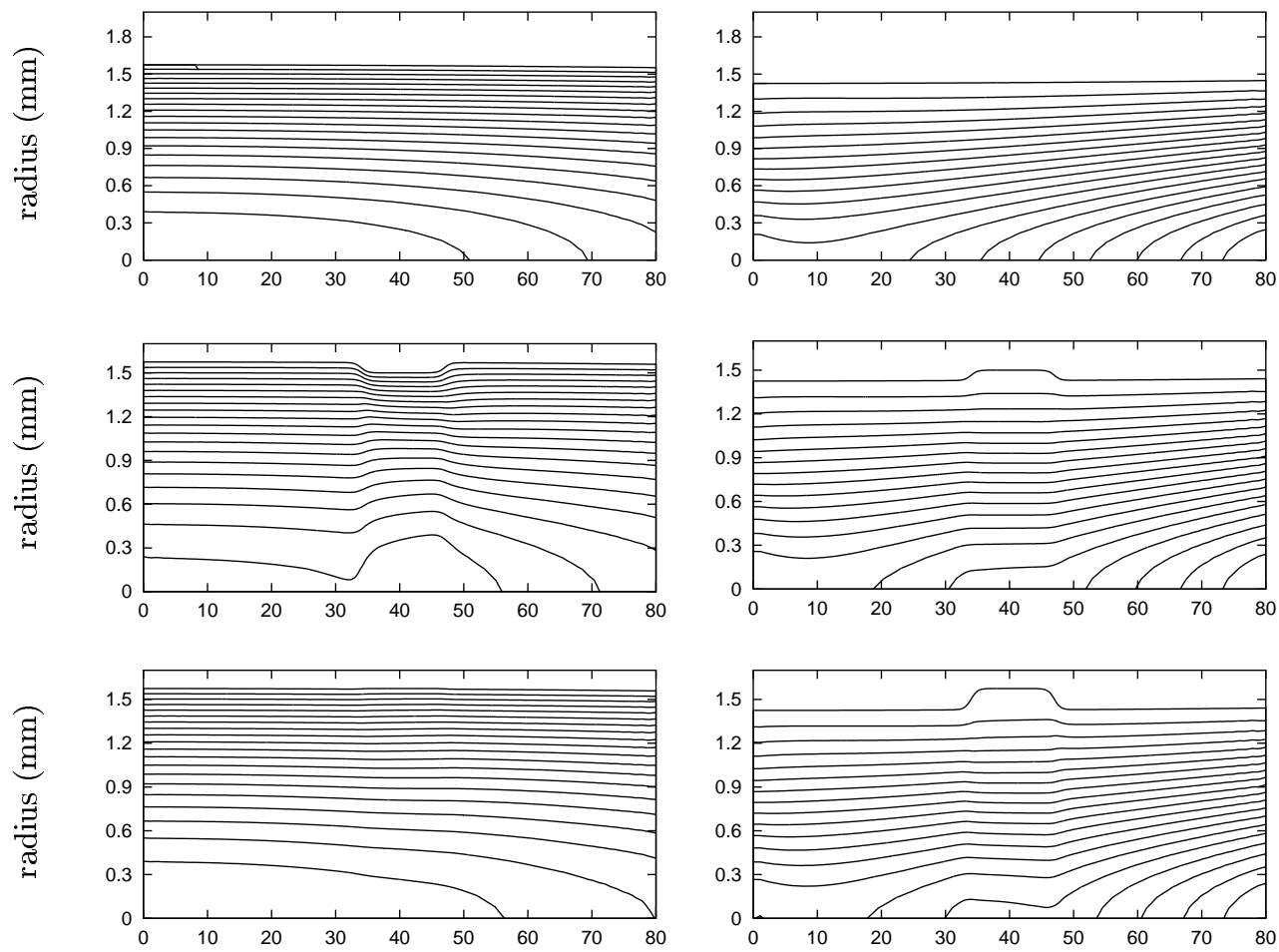

axial position $(\mathrm{mm})$

axial position $(\mathrm{mm})$

FIGURE 5. Shape of the computational domain at times $t=n T$ (systole - left column) and $t=(n+1 / 2) T$ (diastole - right column) for the runs R2 (top row), R3 (medium row) and R4 (bottom row). The isolines of the streamwise velocity are plotted. The streamwise and radial coordinates are expressed in millimetres. The aspect ratio $R_{0} / L$ has been multiplied by 35 for convenience.

\section{Discussion}

Let us first consider the analytical result derived in section 2. A typical value of the speed of propagation of waves in (human coronary) arteries being a few meters per second, the wavelength is usually a few meters. On the other hand, the length scale of the stent is most likely equal to a few centimetres, meaning that the module of the dimensionless parameter $k_{2} L_{2}$ in Eq. (2.4) is small compared to unity. Moreover, since the goal of this study is to assess the wave reflection specific the endovascular prosthesis, one can assume that zero reflection occurs in absence of stent, viz. $A_{1} K_{1}=A_{3} K_{3}$ (in other words, we assume that host artery is perfectly homogeneous). Finally, expanding Eq. (2.4) as a power series of the parameter $k_{2} L_{2}$, one obtains 
the following first order expression for the reflection coefficient:

$$
R_{\text {stent }}=\frac{j\left(1-\Lambda^{2}\right)}{2 \Lambda} k_{2} L_{2}+O\left(k_{2}^{2} L_{2}^{2}\right), \quad \Lambda=\frac{A_{1} K_{1}}{A_{2} K_{2}}
$$

This relation shows that the theoretical reflection induced by an endovascular prosthesis increases with both the pulsation of the wave and the length of the stent. Moreover, it is zero as soon as $\Lambda=1$, viz. $A_{1} K_{1}=A_{2} K_{2}$. Assuming that viscous and viscoelastic effects can be neglected in the reflection process makes $\Lambda$ and $k_{2}$ real numbers with $\Lambda=\sqrt{A_{1} A_{1}^{\prime} / A_{2} A_{2}^{\prime}}$ and $k_{2} L_{2}=\omega \sqrt{\rho A_{2}^{\prime} / A_{2}} L_{2}$. Equation (4.1) then leads to a convenient formula to assess the amount of wave reflection :

$$
R_{\text {stent }} \simeq j \omega \sqrt{\rho} \frac{A_{2} A_{2}^{\prime}-A_{1} A_{1}^{\prime}}{2 \sqrt{A_{1} A_{1}^{\prime}}} \frac{L_{2}}{A_{2}}
$$

From this relation, the amount of wave reflection is related to the geometrical/mechanical mismatch induced by the stenting, a stent shape factor $L_{2} / A_{2}$ as well as the flow conditions. This is coherent with the findings of Formaggia et al. (2001) who solved numerically the 1D non-linear flow equations in a distensible pipe with spatially variable compliance. Since they did not considered harmonic signals, it is difficult to deduce frequency dependant reflection coefficients from their simulations and compare their numerical results with Eq. (4.2). However, in agreement with the present model, they clearly established that the amount of reflection increases with the stent shape factor and compliance mismatch. Finally, Eq. (4.2) shows that a stent satisfying the relation $A_{2}=A_{1} A_{1}^{\prime} / A_{2}^{\prime}$ produces no wave reflection. Clearly, such a prosthesis is not realistic since experimental measurements have shown that $A_{1}^{\prime} / A_{2}^{\prime}$ is as high as 5 (see section 3.1). However, since the compliance $A_{2}^{\prime}$ of the stented artery is always smaller than the compliance $A_{1}^{\prime}$ of the host artery, it follows from Eq. (4.2) that overdilation $\left(A_{2}>A_{1}\right)$ tends to reduce the amount of reflected waves.

Regarding the numerical results of section 3, recall that the objective of the study is to assess the response of blood motion to geometrical perturbations induced by the compliance mismatch 
(itself induced by the presence of the prosthesis). To address this issue, it is relevant to consider the wall motion as an input and to look at modifications in blood flow when stenting is accounting for in the design of this input. Indeed, this approach provides valuable information regarding the hemodynamic changes due to stenting while avoiding to solve the coupled, computer time consuming fluid-structure interaction problem. Still, the design of the input wall motion must be consistent with the effective geometrical changes observed experimentally. Eq. (3.3) has been used in this numerical study to model the wall displacement of an elastic stented artery. It is coherent with the animal experiments (Vernhet et al. 2001) with respect to at least three important aspects of the endovascular stenting:

- it assumes that the speed of propagation within the prosthesis is infinite which is consistent with the fact that the measured compliance at the stent level is several times smaller than its value before/after stenting,

- using the approximation Eq. (4.2) and the numerical values provided in section 3.1, the modulus of the reflection coefficient under in vivo conditions is approximately $\left|R_{\text {stent }}\right| \simeq 2 \%$. It is never more than a few percents if one considers the most unfavourable experimental values in Vernhet et al. (2001). As a result, the pressure field is mostly related to the forward pressure wave, which justifies the fact that no back propagating wave is accounted for in Eq. (3.3).

- although we do not claim that the hyperbolic tangent is the "exact" functional form of the buffer region between the elastic artery and the stent, it is a reasonable candidate for modelling the transition zone. Moreover, the analytical transition length is of order $3 \mathrm{~mm}$ (this is a direct consequence of the damping function $f(x)$ ). This value is in agreement with the observations made during the animal experimentations and showing that the buffer region is close to one diameter long.

Table 2 shows the values of the WSS at the middle of the stent, viz. $x=\left(x_{1}+x_{2}\right) / 2 \simeq 40$ $\mathrm{mm}$. The amplitude of this quantity over the cardiac cycle is larger for the stented vessel than it is for the elastic artery. It is worth noting that although the length of the stent is very small as 
compared to the wavelength, the WSS amplitude in case R3 behaves more like in case R1 (fully rigid tube) and less like in case R2 (elastic tube). The slight overdilation of the prosthesis, by avoiding the increase in shear stress at systole (there is no geometry discontinuity at systole for the case R4, see figure 5), limits drastically the increase in stress amplitude. Figure 4 in section 3.2 suggests that the most important flow changes do not appear near the middle of the stent. This is indeed confirmed by figure 6 where the amplitude of WSS variations over the cycle for cases R3 and R4 is plotted as functions of the axial position. Since these quantities are scaled by their value for the non-stented vessel (case R2), this figure offers a measure of the hemodynamic changes due to the prosthesis in cases R3 and R4. Note that near the stent middle, the values reported in table 2 are recovered. The maximal changes are located slightly downstream the ends of the stent. Near the proximal edge, the WSS variation over the cardiac cycle increases by $50 \%$ in amplitude whereas it decreases by roughly $20 \%$ downstream the distal end of the prosthesis. The modulus of the spatial gradient of the WSS fluctuations is also drastically increased in both edge regions. These findings might be related to the clinical observation that there is a first-order edge effect in some type of restenosis where intimal hyperplasia mostly develops near the stent ends (Weissman et al. 2001). Finally, figure 6 suggests that a slight increase in the stent diameter may reduce drastically the WSS changes induced by stenting. Noticeably, the modifications near the transition regions for the case R4 are smaller than inside the stent.

This theoretical/numerical study supports the idea that stenting can induce endothelial dysfunction via hemodynamic perturbations. Although the amount of pressure reflection is most likely negligible due to the small stent-to-wavelength ratio, the amplitude of the WSS variation over the cardiac cycle is alternatively increased (by $35 \%$ at the middle of the stent, by $50 \%$ in the proximal transition region) and decreased (by $20 \%$ in the distal transition region) by long-standing stenting. Note that the later findings are in good agreement with recent results of Tortoriello \& Pedrizzetti (2004). These authors, by using a perturbative method to solve 


\begin{tabular}{|c|c|c|c|c|}
\hline Run & $\min (\mathrm{WSS})$ & $\max (\mathrm{WSS})$ & $\Delta \mathrm{WSS}$ & $\Delta \mathrm{WSS} / \Delta \mathrm{WSS}_{\mathrm{runR} 2}$ \\
\hline $\mathrm{R} 1$ & 0.39 & 6.72 & 6.33 & 1.29 \\
\hline $\mathrm{R} 2$ & 0.72 & 5.62 & 4.90 & 1.00 \\
\hline $\mathrm{R} 3$ & 0.42 & 6.98 & 6.56 & 1.34 \\
\hline $\mathrm{R} 4$ & 0.22 & 5.66 & 5.44 & 1.11 \\
\hline
\end{tabular}

TABLE 2. Minimum, maximum and (scaled) variations of the wall shear stress (WSS) for different runs at the middle of the stent. Stresses are in Pa.

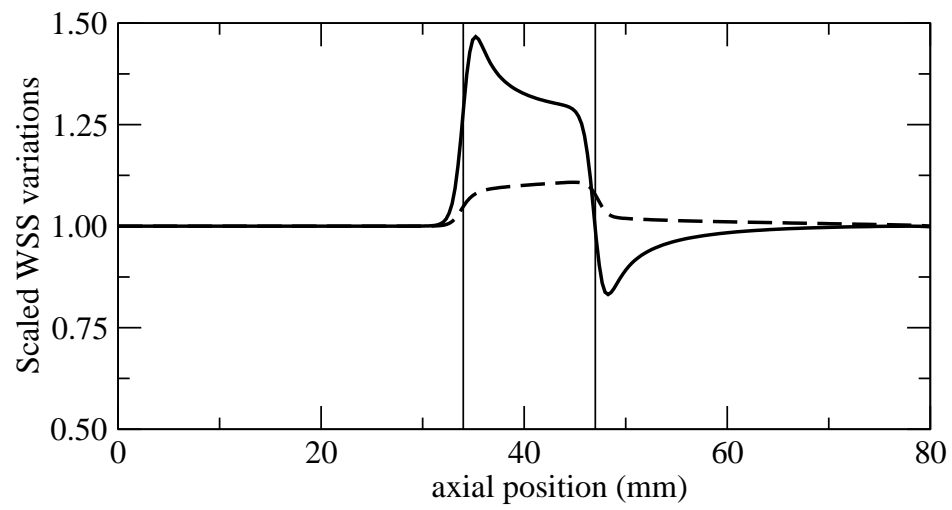

FIgURE 6. Scaled amplitude of the wall shear stress fluctuations for runs R3 (solid) and R4

(long-dashed) The two vertical lines denote the stent location.

the fluid-tissue coupled problem numerically, have shown that stenting decreases the diastolic WSS. They attributed this result to the fact that, during diastole, the artery shrinks more than the stiffer stented region, which is consistent with figure 5 . They also found that the maximum of changes is located near the edges of the stent. However, in contrast to figure 4, they did not observe a large increase of systolic WSS. This seems to be a direct consequence of the geometrical bump that their stented artery experiences during systole at the prosthesis edges. This local deformation, which is related to the particular spatial variations of vessel radius and 
compliance that have been used by Tortoriello \& Pedrizzetti (2004), is not present in our study.

Our results suggest also that over-dilated stents produce less hemodynamic perturbations: (1) from the pressure wave point of view, overdilation tends to lower the amount of reflection, the optimal overdilation being proportional to the compliance ratio. With the typical amount of compliance mismatch observed after stenting (see section 3.1), this result leads to an "optimal" over-expansion close to $600 \%$. Of course this value is unrealistic for clinical applications. It reflects the fact that only acoustic has been accounted for in the 1D model of section 2 and that stenting does not modify the pressure wave propagation drastically: recall that the reflection coefficient is only a few percents because the stent-to-wavelength ratio is always small in clinical applications. (2) the increase of the systo-diastolic WSS fluctuations is only $10 \%$ when the stent radius matches the artery one at systole. Under in vivo conditions, the efficiency of stenting does not depend only on hemodynamics and other effects must be accounted for. Regarding stent overdilation, an increased intimal hyperplasia is always demonstrated (Vernhet et al. 2003; Schwartz et al. 1992) despite contradictory clinical consequences (Caixeta et al. 2000; Koyama et al. 2000) are reported in the literature. However, it is likely that an optimum overdilation rate exists, which limits the amount of hemodynamic perturbations without inducing unacceptable vascular stretching. Further studies are necessary to clarify this issue.

\section{Conclusion}

Two simple models have been developed and used in order to study the changes in blood motion due to long-standing stenting. The details of the stent structure were not accounted for because the objective was to focus on the mid-term (after the wires have been integrated with the surrounding tissue) effects of stenting on hemodynamics. Accordingly, the prosthesis was modelled as a uniform rigid tube embedded in an elastic artery and the effects of the compliance mismatch have been studied.

The first model is fully analytical and allow to assess the amount of pressure wave reflection 
due to stenting under the $1 \mathrm{D}$ assumption. An easy-to-use formula is provided in order to assess the reflection coefficient from the knowledge of the compliance before and after stenting. It is found that it is proportional to the stent-to-wavelength ratio and that the amount of wave reflection remains rather small in clinical applications. This result is the basis of the second numerical model developed in order to assess the wall shear stress changes. In this approach, the fully coupled fluid-structure problem is replaced by a simpler fluid problem with moving boundaries. The amount of pressure reflection being small, a simple analytical law can be used to describe the wall motion over space and time. The Navier-Stokes equations are then solved numerically with an appropriate finite element based method which handles time dependent geometries. The main result is that, due to stenting and the corresponding compliance mismatch, the amplitude of the wall shear stress variations is drastically increased, by more than $30 \%$ at the middle of the stent, more than $50 \%$ in the proximal transition region. These results support the idea that stenting can induce endothelial dysfunction via hemodynamic perturbations. They are also consistent with the fact that intimal hyperplasia is often more developed near the edges of the stents. Finally, our numerical simulations indicate that the negative effects of long-standing stenting on hemodynamics decrease when the radius of the prosthesis is sligthly increased.

\section{REFERENCES}

Bénard, N., Coisne, D. \& Perrault, R. 2003 Simulation of blood flow in stented artery: Blood rheological properties effects. In XXVIII Congrès, Poitiers, France. Société de Biomécanique.

Berry, J., Santamarina, A., Moore, J., Roychowdhury, S. \& Routh, W. 2000 Experimental and computational flow evaluation of coronary stents. Annals of Biomedical Engineering 28, 386-398.

Caixeta, A., Brito, F. J. \& Rati, M. 2000 High versus low-pressure balloon inflation during multilinktrade mark stent implantation: acute and long-term angiographic results. Catheter Cardiovasc. Interv. 50, 398-401.

Caro, C., Fitz-Gerald, J. \& Schroter, R. 1969 Arterial wall shear and distribution of early atheroma in man. Nature 211 (223), 1159-1160. 
Chorin, J. 1967 A numerical method for solving incompressible viscous flow problems. Journal of Computational Physics 2, 12-26.

Davies, P., Shi, C., DePaola, N., Helmke, B. \& Polacek, D. 2001 Hemodynamics and the focal origin of atherosclerosis. a spatial approach to endothelial structure, gene expression, and function. Ann. N.Y. Acad. Sci. 947, 7-16.

Formaggia, L., Nobile, F. \& Quarteroni, A. 2001 A one dimensional model for blood flow: application to vascular prosthesis. In In MSCOM2000, edited by T. Miyoshi, Springer-Verlag, Berlin.

Grube, E., Silber, S., Hauptmann, K., Mueller, R., Buellesfeld, L., Gerckens, U. \& RusSELL, M. 2003 Taxus i: six- and twelve-month results from a randomized, double-blind trial on a slow-release paclitaxel-eluting stent for de novo coronary lesions. Circulation 107 (1), 38-42.

Hughes, T. \& Lubliner, J. 1973 On the 1D theory of blood flow in the larger vessels. Math. Biosciences 18, 161-170.

Koyama, J., Owa, M. \& Sakurai, S. 2000 Relation between vascular morphologic changes during stent implantation and the magnitude of in-stent neointimal hyperplasia. Am. J. Cardiol. 86, $753-758$.

Medic, G. \& Mohammadi, B. 1999 Nsike - an incompressible navier-stokes solver for unstructured meshes. INRIA - Research Report 3644 .

Morice, M., Serruys, P., Sousa, J., Fajadet, J., Ban Hayashi, E., Perin, M., Colombo, A., Schuler, G., Barragan, P., Guagliumi, G., Molnar, F. \& Falotico, R. 2002 Ravel study group. randomized study with the sirolimus-coated bx velocity balloon-expandable stent in the treatment of patients with de novo native coronary artery lesions. a randomized comparison of a sirolimus-eluting stent with a standard stent for coronary revascularization. $N$ Engl $J$ Med 346 (23), 1773-1780.

Nicoud, F. 2002 Hemodynamic changes induced by stenting in elastic arteries. Center for Turbulence Research, CTR Annual Research Briefs pp. 336-347.

Rau, T., Schofer, J., Schluter, M., Seidensticker, A., Berger, J. \& Mathey, D. 1998 Stenting of non acute total coronary occlusions: predictors of late angiographic outcome. J. Am. Coll. Card. 31, $275-280$.

Reuderink, P., Hoogstraten, H., Sipkema, P., Hillen, B. \& Westerhof, N. 1989 Linear and nonlinear one-dimensional models of pulse wave transmission at high womersley numbers. $J$. Biomech. 22, 819-827. 
Robinson, K., Roubin, G., Siegel, R., Black, A., Apkarian, R. \& King, S. 1988 Intra-arterial stenting in the atherosclerotic rabbit. Circulation 78, 646-653.

Rolland, P., Charifi, A. \& Verrier, C. 1999 Hemodynamics and wall echanics after stent placement in swine illiac arteries: comparative results from six stent designs. Radiology 213, 229-246.

Schwartz, R., Huber, K. \& Murphy, J. 1992 Restenosis and the proportional neointimal response to coronary artery injury: results in a porcine model. J. Am. Coll. Cardiol. 19, 267-274.

Serruys, P., Degertekin, M., Tanabe, K., Abizaid, A., Souza, J., Colombo, A., Guagliumi, G., Wijns, W., Lindeboom, W., Ligthart, J., De Feyter, P. \& Morice, M. 2002 Intravascular ultrasound findings in the multicenter, randomised, double-blind ravel (randomised study with the sirolimus-eluting velocity balloon-expandable stent in the treatment of patients with de novo native coronary artery lesions) trial. Circulation 106, 798-803.

Tortoriello, A. \& Pedrizzetti, G. 2004 Flow-tissue interaction with compliance mismatch in a model stented artery. Journal of Biomechanics 37, 1-11.

Vernhet, H., Demaria, R., Oliva-Lauraire, M., Juan, J., SÉnac, J. \& Dauzat, M. 2001 Changes in wall mechanics after endovascular stenting in rabbit aorta: comparison of three different stent designs. Am. J. Roent. 176 (3), 803-807.

Vernhet, H., Demaria, R., Pérez-Martin, A., Juan, J., Oliva-Lauraire, M., Marty-Double, C., , SÉnac, J. \& Dauzat, M. 2003 Wall mechanics of the stented rabbit aorta: Long-term study and correlation with histological findings. J. Endovascular Therapy 10 (3), 577-584.

Vernhet, H., Juan, J., Demaria, R., Oliva-lauraire, M., SÉnac, J. \& Dauzat, M. 2000 Acute changes in aortic wall mechanical properties after stent placement in rabbits. J. Vasc. Inter. Rad. 11 (5), 634-638.

Weissman, N., Wilensky, R., Tanguay, J., Bartorelli, A., Moses, J., Williams, D., Bayley, S., Martin, J., Canos, M., Rudra, H., Popma, J., Leom, M., Kaplan, A. \& Mintz, G. 2001 Extent and distribution of in-stent intimal hyperplasia and edge effect in a non-radiation stent population. Am J Cardiol 88, 248-252.

Womersley, J. 1955 Oscillatory motion of a viscous liquid in a thin-walled elastic tube- i: The linear approximation for long waves. Philosophical Magazine (series 7) 46, 199-221. 